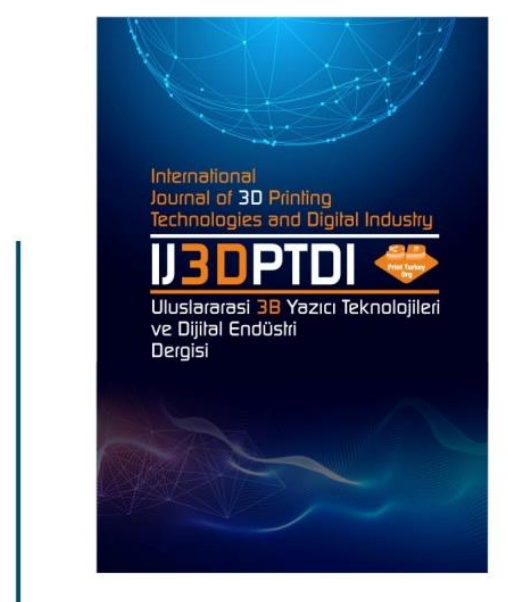

ULUSLARARASI 3B YAZICI TEKNOLOJILERI

VE DIJITAL ENDÜSTRI DERGISI

INTERNATIONAL JOURNAL OF 30 PRINTING TECHNQLOGIES AND DIGITAL INDUSTRY

I55N:2602-3350 [Online]

URL: https://dergipark.org.tr/ij3dptdi

\title{
THE EFFECT OF TRIACRYLATE MONOMER STRUCTURE ON VOLUME SHRINKAGE AND TENSILE PROPERTIES OF VAT POLYMERIZATION RESINS
}

Yazarlar (Authors): Volkan Kılıç, (i)*, Şebnem Camadanlı

Bu makaleye şu şekilde atıfta bulunabilirsiniz (To cite to this article): Kılıç V., Camadanlı Ş. "The Effect of Triacrylate Monomer Structure on Volume Shrinkage and Tensile Properties of VAT Polymerization Resins" Int. J. of 3D Printing Tech. Dig. Ind., 5(2): 220-226, (2021). 


\title{
THE EFFECT OF TRIACRYLATE MONOMER STRUCTURE ON VOLUME SHRINKAGE AND TENSILE PROPERTIES OF VAT POLYMERIZATION RESINS
}

\author{
Volkan Kılıça, iD*, Şebnem Camadanla ${ }^{\text {i }}$ \\ ${ }^{\text {a }}$ Kempropol Kimyasal ve Polimer Maddeler San. ve Tic. A.Ş. \\ *Corresponding Author: volkankilic@kempro.com.tr
}

(Received: 16.06.2021; Revised: 26.07.2021; Accepted: 04.08.2021)

\begin{abstract}
This study investigates the influence of triacrylate monomer structure on volume shrinkage, tensile properties, and viscosity of vat polymerization (VP) resins. The amount of triacrylate monomer kept constant as $20 \%$ wt. to prevent excessive volume shrinkage effect. Results indicated that ethoxylated and propoxylated triacrylate structures are beneficial to reduce the volume shrinkage of VP resins. However, these flexible chain structures led to a reduction in tensile properties and the elastic modulus of VP resins deteriorated up to 35\% compared to trimethylolpropane triacrylate (TMPTA). There is no significant effect observed on VP resin reactivity according to the triacrylate type. Besides, ethoxylated triacrylate presented the best dilution effect on VP resin.
\end{abstract}

Keywords: VAT Polymerization, UV-curable resins, SLA, acrylate, photopolymerization, 3D Printing

\section{INTRODUCTION}

Three-dimensional (3D) printing is a method to produce solid elements based on the gradual assembling of materials. 3D printing is also called additive manufacturing (AM) or rapid manufacturing [1-2]. The history of AM dates back to the 1960s but the first commercial use of AM was realized by stereolithography (SLA) 3D printer from 3D Systems in 1987 [3]. After this milestone, several AM methods were developed and launched to the market. AM technologies were classified by the "American Society for Testing and Materials" (ASTM) [4] and classification is shown in Figure 1.

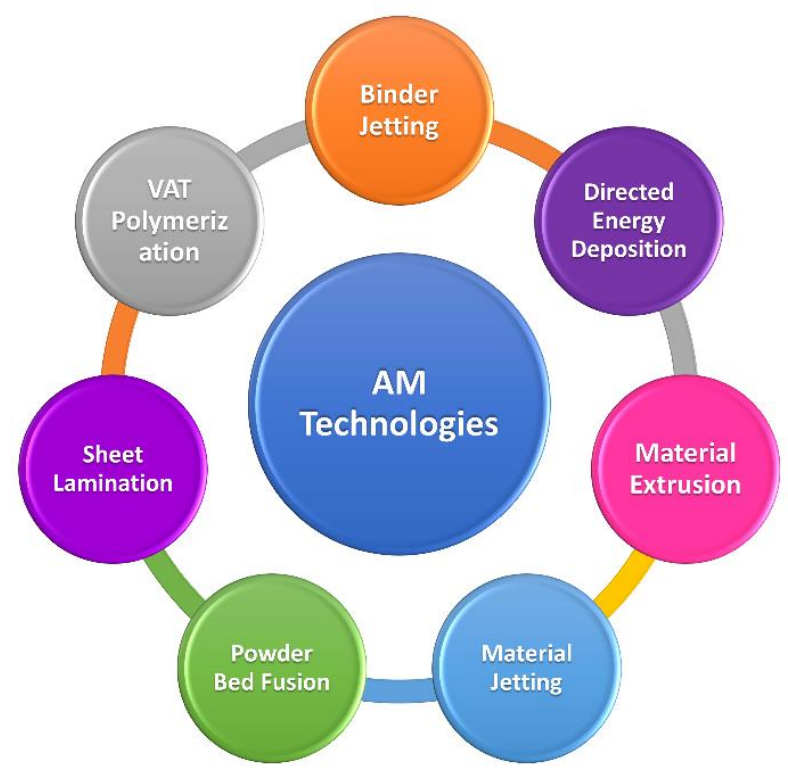

Figure 1. Types of 3D Printing Technologies (ASTM F2792-12a) 
VAT polymerization (VP) is an additive manufacturing method, based on gradual solidification of photocurable resin material. In addition to the aforementioned classification of AM technologies, VP methods can be divided into three types; "laser stereolithography" (SLA), "digital light processing" (DLP), and "liquid crystal display" (LCD) [5]. Although all VP methods are following the same principles, they are differentiated according to the type of UV light source. SLA 3D printers utilize a UV laser and galvanometer to scan and cure each layer. The precision of the 3D printed part is determined by the beam size of the laser. DLP 3D printers utilize a UV light source created by a digital projector. A layer cured in one illumination and precision of the is part adjusted by projector's resolution. An LED array is used in LCD 3D printers as a UV light source. At this method, an LCD screen masks the UV light to cure the individual layer. The quality of the final part is directly related to the resolution of the LCD screen. Schematic representation of VP methods is shown in Figure 2.

\section{SLA}

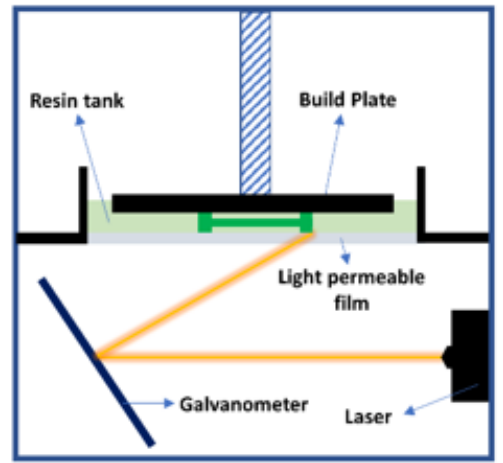

DLP

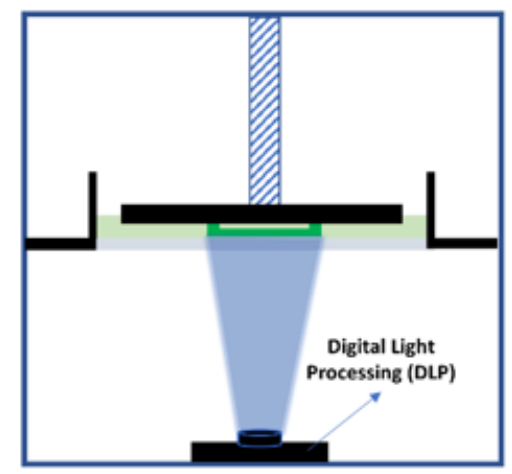

LCD

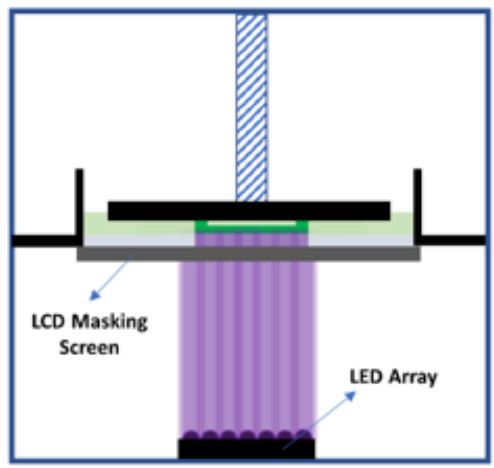

Figure 2. Schematic Representation of VP methods

VP resins are commercially used in numerous applications such as jewelry modeling [6-7], automotive part prototyping [8-11], dental modeling [12], footwear modeling [13-14], and so on. Simultaneously, researchers make an extensive effort to develop self-healing [15-16], shape-memory [17-18], and biobased [19-21] VP resins. VP resins can be formulated by free radical curable (meth)acrylic materials [22-24], cationic curable epoxy-functional materials [25] or both free radical and cationic curable (meth)acrylate/epoxy mixtures [26]. In this article, (meth)acrylate-based VP resin is formulated. Acylate-based VP resins are the composition of (meth)acrylic monomers, (meth)acrylic oligomers, free radical photo-initiators, stabilizers, light absorbers, and other additives [27]. Acrylate-based resins exhibited high reactivity and fast curing compared to epoxy-based resins. However, acrylate-based presented high-volume shrinkage by contrast with epoxy-based resins [28].

One of the most common approaches to reduce volume shrinkage is the mixing of epoxy-based and acrylate-based resins. Shan et al. [29] reduced the volume shrinkage of 3D printed parts from $10.44 \%$ to $7.41 \%$ with the addition of epoxy resin. However, a high amount of epoxy resin led to a reduction of mechanical properties before post-curing. Besides, additional thermal post-curing is essential to cure the epoxy part of the resin mixture. Deng et al. [30] concluded that volume shrinkage of acrylate-based resins increases with the amount of monofunctional reactive diluent. Besides, acrylate oligomer content exhibited the opposite effect and led to the reduction of volume shrinkage. However high oligomer content caused the high viscosity and low reactivity.

Three functional acylate monomers are widely used as a crosslinker for acrylate-based VP resins. On the other hand, a high amount of these acrylates may lead to a significant amount of volume shrinkage. In this article, the effect of the type of three functional acrylate monomer on volume shrinkage is investigated. 


\section{MATERIALS AND METHOD}

Trimethylolpropane triacrylate (TMPTA, commercial name Miramer M300) and Ethoxylated (3) Trimethylolpropane Triacrylate (EO3, commercial name Miramer M3130) were supplied by Miwon Specialty Chemicals (Ansan, South Korea). Propoxylated (3) Trimethylolpropane Triacrylate (PO3, commercial name SR492) was kindly provided by Sartomer-Arkema (Colombes Cedex, France). Figure 3. shows the chemical structures of triacrylate monomers. Isopropyl alcohol (IPA, technical grade) was purchased by Kempro Kimya, (İstanbul, Turkey).

Table 1. Formulations of VP resins.

\begin{tabular}{lcccc}
\hline \hline Abbreviations & $\begin{array}{c}\text { TMPTA } \\
(\boldsymbol{\%} \text { wt. })\end{array}$ & $\begin{array}{c}\text { EO3 } \\
(\boldsymbol{\%} \text { wt. })\end{array}$ & $\begin{array}{c}\text { PO3 } \\
(\boldsymbol{\%} \text { wt. })\end{array}$ & $\begin{array}{c}\text { Other Components* } \\
\text { (\% wt. })\end{array}$ \\
\hline \hline TMPTA & 20 & - & - & 80 \\
EO3 & - & 20 & - & 80 \\
PO3 & - & - & 20 & 80 \\
\hline \hline
\end{tabular}

"Other components are the mixture of acrylic monomers, oligomers, and a photoinitiator.

VP resin formulations are shown in Table 1 and prepared by prior mixing of urethane acrylate oligomers and acrylic monomers that have mono, di, or trifunctional groups at $50^{\circ} \mathrm{C}$ with a magnetic stirrer. After that, a phosphine oxide type photoinitiator (PI) was added and mixed until it completely dissolved.

"Anycubic Photon S LCD 3D printer" was utilized to produce test specimens which has a 405nm 50W UV LED array and a $2560 \times 1440$ (2K) HD LCD screen. 3D printed test specimens were washed with IPA and post-cured for 6 minutes via "Anycubic Wash\&Cure Machine" which has 365 and 405nm 40W UV LED array with a rotatable platform to finalize the curing of 3D printed parts.

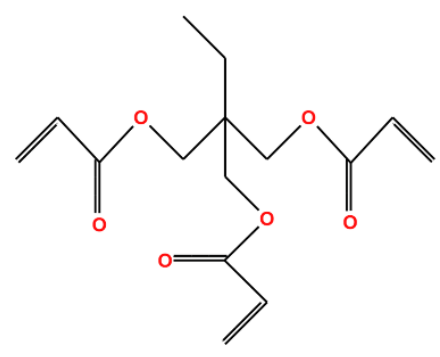

(a)

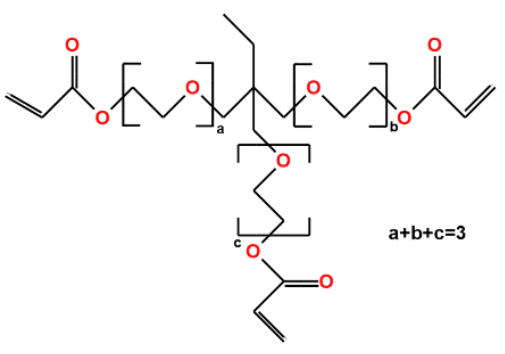

(b)

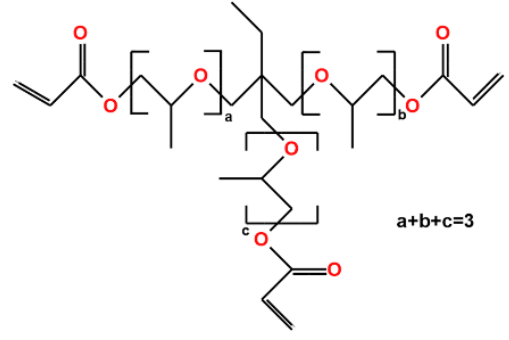

(c)

Figure 3. Chemical structures of triacrylate monomers, (a) Trimethylolpropane triacrylate (TMPTA), (b) Ethoxylated (3) Trimethylolpropane Triacrylate (EO3), (c) Propoxylated (3) Trimethylolpropane Triacrylate (PO3).

The tensile test was applied by using Devotrans GPSP/R Universal Tensile Tester according to the ASTM D638 with specimen type "V" as mentioned by Saraç et. al. [31] and $1 \mathrm{~mm} / \mathrm{min}$ test speed. Tensile test specimens were produced with $0.05 \mathrm{~mm}$ layer height. Layer curing time was set to 60 seconds for 10 layers and 10 seconds for the following layers.

The viscosity of VP resins was measured using Brookfield CAP $2000+$ Cone\&Plate Viscometer at $25^{\circ} \mathrm{C}$. Volume shrinkage was calculated according to equation (1) where $\rho_{\text {liquid }}$ and $\rho_{\text {solid }}$ are the density of the VP resins before and after 3D printing, respectively. Figure 4. Shows the Anycubic Photon S LCD 3D Printer and the Anycubic Wash\&Cure Machine.

$$
\text { Volume shrinkage }(\%)=\frac{\rho_{\text {solid }}-\rho_{\text {liquid }}}{\rho_{\text {solid }}} \times 100
$$

Solid density was measured by using Mettler Toledo Precision Balance XPR204S Lab Scale equipped with a density kit. Liquid density was measured by using a pycnometer. Resin reactivity test applied using Photon S 3D printer. Firstly, the resin tank was taken off and a clear PET sheet was placed onto the LCD screen. Then, 2-3 grams of VP resin were poured onto the film. Screen activated 10 seconds 
using exposure test mode. The uncured resin was washed with IPA and cured film peeled off from the PET sheet. Cured film thickness was measured by using a micrometer.

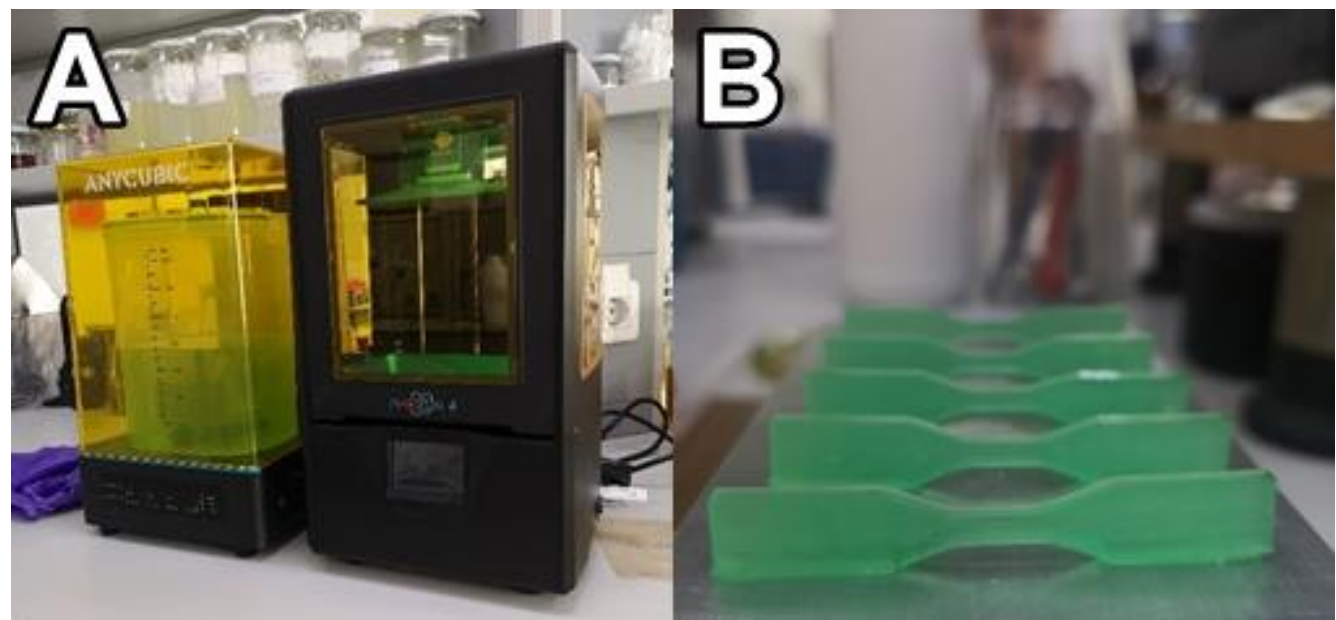

Figure 4. (A) “Anycubic Photon S 3D Printer" (right) and "Anycubic Wash\&Cure Machine" (left) and (B)

Tensile test specimens

\section{RESULTS AND DISCUSSION}

Before the production of test specimens, the reactivity of the VP resins was analyzed. Cured film thicknesses of VP resins were determined as $0.21 \mathrm{~mm}$ according to the aforementioned method. Results indicated that the reactivity of VP resins was not affected significantly by the type of triacrylate monomer for $20 \%$ wt. addition. However, the reactivity of VP resins may differ for higher triacrylate monomer content due to the excessive crosslinking effect of the trifunctional monomers.

Figure 5. shows the viscosities of VP resins. TMPTA and PO3 exhibited the same viscosity while EO3 presenting a lower value. TMPTA and PO3 have the same dilution effect on VP resins. On the other hand, EO3 shows higher dilution efficiency, in other words, higher cutting power. EO3 may be led to better dilution owing to its flexible ethoxylated chemical structure.

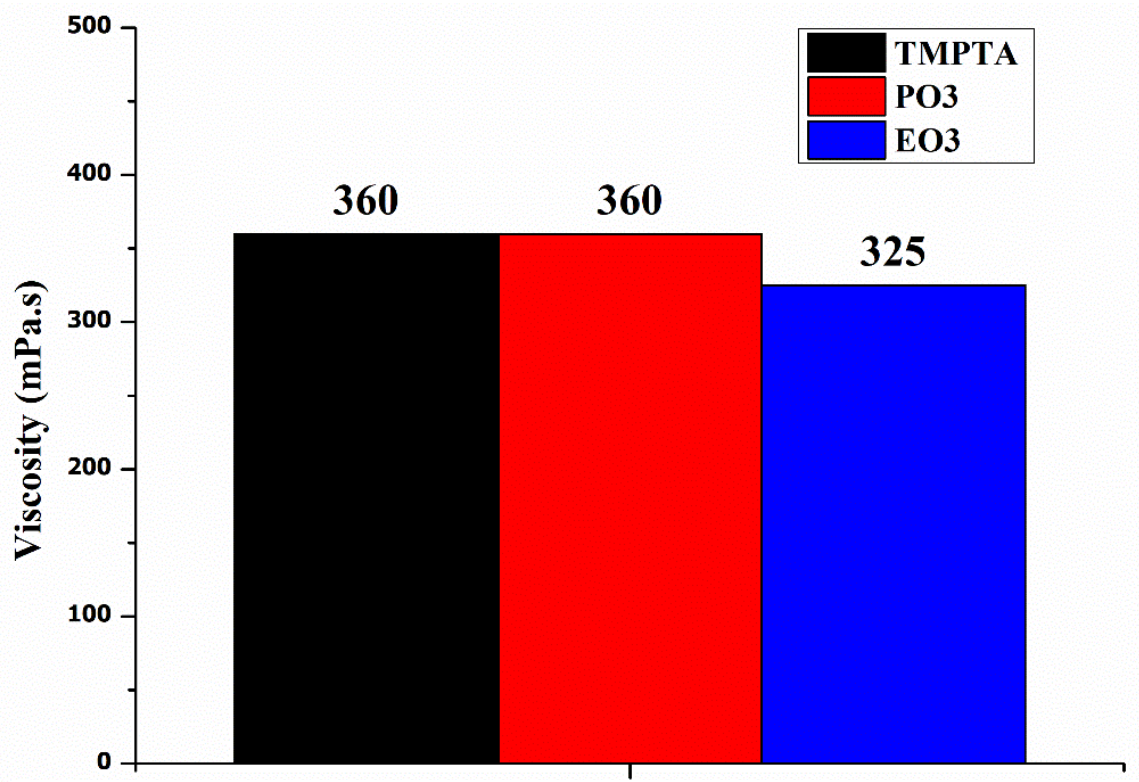

Figure 5. Cone\&Plate viscosities of VP resins.

Figure 6. shows the tensile test results. TMPTA showed the highest stiffness compared to EO3 and PO3. The elastic modulus of TMPTA is almost 1.5 times higher than others. On the other hand, EO3 and PO3 exhibited almost 2 times higher elongation compared to TMPTA. Ethoxylated and propoxylated 
triacrylates contributed to the elasticity of VP resins owing to their flexible chemical structures while TMPTA providing rigidity and stiffness.

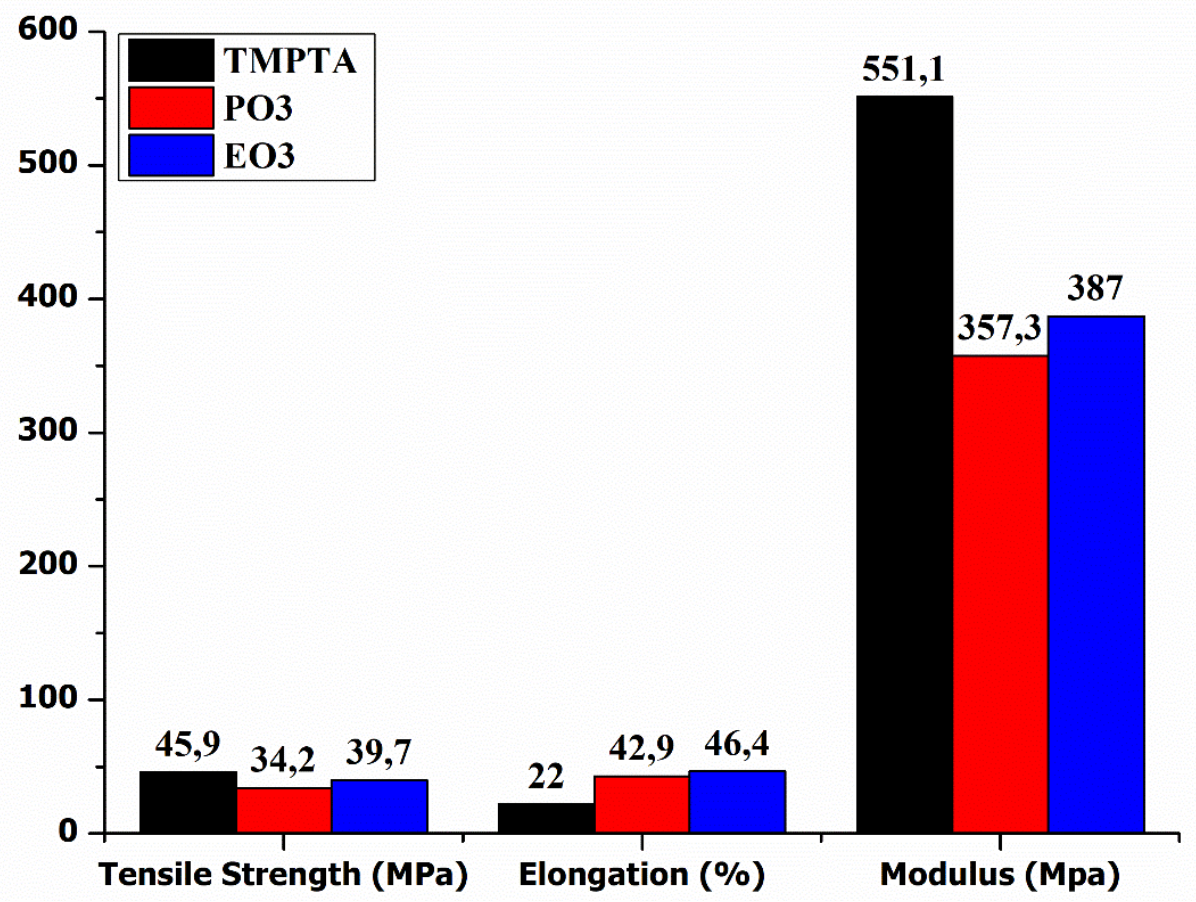

Figure 6. Tensile Properties of VP Resins

Table 2. shows the density and volume shrinkage values of VP resins. Solid densities of VP resins varied prominently compared to liquid densities. Thus, the volume shrinkage of VP resins differentiated according to the triacrylate structure. PO3 led to the lowest volume shrinkage while TMPTA presenting the highest volume shrinkage. Ethoxylated and propoxylated structures may limit the close packing of polymer chains after curing and lead to a decrease in shrinkage. This phenomenon may be observed more significantly for PO3 compared due to its spacer pendant propyl group.

Table 2. Density and volume shrinkage values of VP resins

\begin{tabular}{lccc}
\hline \hline Abbreviations & $\begin{array}{c}\boldsymbol{\rho}_{\text {solid }} \\
\left(\mathbf{g} / \mathbf{c m}^{\mathbf{3}}\right)\end{array}$ & $\begin{array}{c}\text { Pliquid } \\
\left(\mathbf{g} / \mathbf{c m}^{\mathbf{3}}\right)\end{array}$ & $\begin{array}{c}\text { Volume Shrinkage } \\
(\boldsymbol{\%})\end{array}$ \\
\hline \hline TMPTA & 1.1836 & 1.0882 & 8.06 \\
EO3 & 1.1762 & 1.0880 & 7.49 \\
PO3 & 1.1686 & 1.0885 & 6.85 \\
\hline
\end{tabular}

\section{CONCLUSION}

The effect of triacrylate type on the volume shrinkage, viscosity, and tensile properties was investigated in this article. Results concluded that triacrylate type has a significant effect on final VP resin properties except for the reactivity. Ethoxylated and propoxylated groups promote flexibility by increasing the elongation at break up to $210 \%$ and reduce shrinkage from $8,06 \%$ up to $6,85 \%$. However, ethoxylated and propoxylated groups led to a dramatic reduction in elastic modulus up to $35 \%$ for $\mathrm{PO} 3$ compared to TMPTA. Mixtures of triacrylate monomers can be beneficial to obtain balanced results of volume shrinkage and elastic modulus.

\section{REFERENCES}

1. Sachs, E., Cima, M., \& Cornie, J., "Three-dimensional printing: rapid tooling and prototypes directly from a CAD model", CIRP Annals, Vol. 39, Issue 1, Pages 201-204, 1990. 
2. Kruth, J. P., Leu, M. C., \& Nakagawa, T., "Progress in additive manufacturing and rapid prototyping.", Cirp Annals, Vol. 4, Issue 2, Pages 525-540, 1998.

3. Wohlers "History of Associates, Additive http://www.wohlersassociates.com/history2014.pdf, August 10, 2021.

4. Standard, A. S. T. M., "Standard terminology for additive manufacturing technologies." ASTM International F2792-12a, 2012.

5. Fiedor, P., \& Ortyl, J., "A new approach to micromachining: High-precision and innovative additive manufacturing solutions based on photopolymerization technology", Materials, Vol. 13, Issue 13, 2951, 2020.

6. Envisiontech 3D Printing Materials, "Jewelry", https://envisiontec.com/3d-printing-materials/jewelry/, August 10, 2021.

7. Formlabs 3D Printing Materials, "How 3D Printing is Disrupting the Jewelry Industry", https://formlabs.com/blog/3d-printed-jewelry/, August 10, 2021.

8. 3dprint.com, "Lamborghini Rolls out Sián Roadster with 3D Printed Air Vents" https://tinyurl.com/hyam4nhk, August 10, 2021.

9. 3dprintingindustry.com, "Ford Opens Advanced Manufacturing Center and 3D Prints Ford Shelby Mustang GT500 Parts", https://tinyurl.com/3975xz3s, August 10, 2021.

10. RPS 3D Printers, "Williams Racing invest big with RPS” https://tinyurl.com/4kbkj4dy, August 10, 2021.

11. RPS 3D Printers, "BAC utilises British 3D printing companies RPS and Malcolm Nicholls Limited to produce 3D printed parts for new Mono R supercar", https://tinyurl.com/37tm8ndt, August 10, 2021.

12. decisionsindentistry.com, "Stereolithography: The Future of Dental Fabrication" https://tinyurl.com/552mr2ce, August 10, 2021.

13. amfg.ai, “Application Spotlight: 3D Printing for Footwear”, https://tinyurl.com/3sc4jvey, August 10, 2021.

14. RPS 3D Printers, "Clarks Shoes, shaping future footwear development and innovation with 3D printing and RPS” https://tinyurl.com/587jcjar, August 10, 2021.

15. Li, X., Yu, R., He, Y., Zhang, Y., Yang, X., Zhao, X., \& Huang, W., "Self-healing polyurethane elastomers based on a disulfide bond by digital light processing 3D printing", ACS Macro Letters, Vol. 8, Issue 11, Pages 1511-1516, 2019.

16. Wallin, T. J., J. H. Pikul, S. Bodkhe, B. N. Peele, B. C. Mac Murray, D. Therriault, B. W. McEnerney, R. P. Dillon, E. P. Giannelis, and R. F. Shepherd, "Click chemistry stereolithography for soft robots that self-heal", Journal of Materials Chemistry B, Vol.5, Issue 31, Pages 6249-6255, 2017.

17. Choong, Y. Y. C., Maleksaeedi, S., Eng, H., Wei, J., \& Su, P. C. (2017). 4D printing of high-performance shape memory polymer using stereolithography. Materials \& Design, 126, 219-225, 2017.

18. Zhao, Tingting, Ran Yu, Xinpan Li, Bing Cheng, Ying Zhang, Xin Yang, Xiaojuan Zhao, Yulei Zhao, Wei Huang. "4D printing of shape memory polyurethane via stereolithography." European Polymer Journal, Vol. 101, Pages 120-126, 2018.

19. Voet, V. S., Strating, T., Schnelting, G. H., Dijkstra, P., Tietema, M., Xu, J., Folkersma, R., "Biobased acrylate photocurable resin formulation for stereolithography 3D printing" ACS Omega, Vol. 3, Issue 2, Pages 1403-1408, 2018.

20. Sutton, J. T., Rajan, K., Harper, D. P., \& Chmely, S. C., "Lignin-containing photoactive resins for 3D printing by stereolithography" ACS applied materials \& interfaces, Vol. 10, Issue 42, Pages 36456-36463, 2018. 
21. Guit, J., Tavares, M. B., Hul, J., Ye, C., Loos, K., Jager, J., Voet, V. S., "Photopolymer resins with biobased methacrylates based on soybean oil for stereolithography" ACS Applied Polymer Materials, Volume 2, Issue 2, Pages 949-957, 2020.

22. Borrello, J., Nasser, P., Iatridis, J. C., Costa, K. D., “3D printing a mechanically-tunable acrylate resin on a commercial DLP-SLA printer", Additive manufacturing, Vol. 23, Pages 374-380, 2018.

23. Park, H. K., Shin, M., Kim, B., Park, J. W., Lee, H., “A visible light-curable yet visible wavelengthtransparent resin for stereolithography 3D printing”, NPG Asia Materials, Vol. 10, Issue 4, Pages 82-89, 2018.

24. Saraç, M. F., Merve, M., Bülbül, İ., Aktitiz, İ., Yalçın, B. S., Varol, R., "Stereolitrografi ile 3B Basılabilir Nanokil Takviyeli Polimer Yapıların Mekanik Karakterizasyonu", Journal of the Institute of Science and Technology, Volume 9, Issue 3, Pages 1584-1593. 2019.

25. Huang, B., Wu, B., Han, L., Lu, Z., Zhou, W., "Preparation of a novel cationic photosensitive resin (3DSLR01) for stereolithography 3D printing and determination of its some properties", Journal of Wuhan University of Technology-Material Science Ed., Vol. 34, Issue 4, Pages 761-768, 2019.

26. Li, J., Wang, L., Dai, L., Zhong, L., Liu, B., Ren, J., Xu, Y., "Synthesis and characterization of reinforced acrylate photosensitive resin by 2-hydroxyethyl methacrylate-functionalized graphene nanosheets for 3D printing”, Journal of Materials Science, Vol. 53, Issue 3, Pages 1874-1886, 2018.

27. Ligon, S. C., Liska, R., Stampfl, J., Gurr, M., Mülhaupt, R., "Polymers for 3D printing and customized additive manufacturing", Chemical reviews, Vol. 117, Issue 15, Pages 10212-10290, 2017.

28. Schmidleithner, C., Kalaskar, D. M., "Stereolithography”, Cvetković, D., 3D Printing, Pages 1-22, Intech Open, London, 2018.

29. Shan, J., Yang, Z., Chen, G., Hu, Y., Luo, Y., Dong, X., Zhou, W., "Design and Synthesis of FreeRadical/Cationic Photosensitive Resin Applied for 3D Printer with Liquid Crystal Display (LCD) Irradiation”, Polymers, Vol. 12, Issue 6, Pages 1346, 2020.

30. Deng, Y., Li, J., He, Z., Hong, J., Bao, J., "Urethane acrylate-based photosensitive resin for three-dimensional printing of stereolithographic elastomer", Journal of Applied Polymer Science, Vol. 137, Issue 42, Pages 49294, 2020.

31. Saraç, M. F., Oranlı, A., Aktitiz, İ., Yalçın, B. S., Varol, R., “3B Basılabilir Füme Silika Takviyeli FotoDuyarlı Polimerlerin Mekanik Özelliklerinin İncelenmesi” Düzce Üniversitesi Bilim ve Teknoloji Dergisi, Volume 7, Issue 3, Pages 1793-1805, 2019. 